\title{
Gait assessment in general practice
}

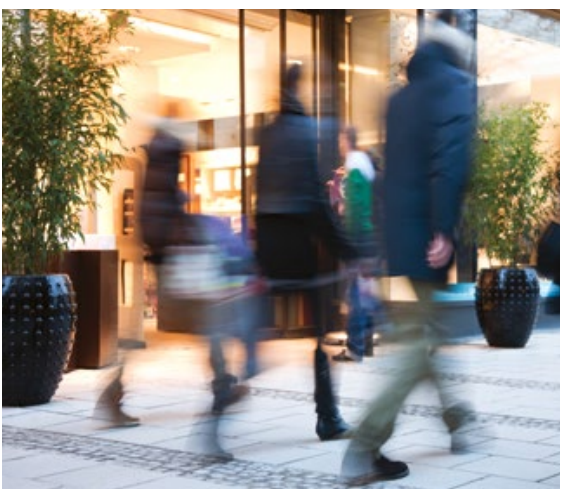

CPD

Appukutty Manickam, Matthew D Gardiner

\section{Background}

Patients with a variety of neuromuscular pathologies may present with gait abnormalities. Accurate assessment of each pattern, coupled with appropriate investigations, facilitates diagnostic accuracy followed by treatment or referral, as required.

\section{Objective}

This review outlines the relevant history and examination areas to assess when interpreting gait abnormalities, together with common gait patterns and their aetiologies.

\section{Discussion}

A range of factors should be evaluated, including posture, walking aids and footwear. The gait cycle can be broadly divided into the stance and swing phases, and additional features are assessed to identify specific patterns and generate a provisional diagnosis. Gait examination, from the time the patient is called from the waiting room, will help the clinician focus on the relevant systems to examine, investigate and treat.
мовILITY has a disproportionate impact on independence and function, as walking underpins most other activities of daily living. The lower extremity is dedicated to the vital tasks of weight bearing and ambulation. ${ }^{1}$ There is debate regarding how, why and when human bipedalism evolved, with estimates for the latter ranging from four to seven million years ago. Each person's gait pattern is unique; however, there are often recognisable familial elements.

There are two main phases in the normal walking cycle: 1 ) the stance phase, when the foot is on the ground, and 2) the swing phase, when the foot is moving forward. ${ }^{1}$ Sixty per cent of the normal cycle is spent in the stance phase (25\% of the cycle in double stance, with both feet on the ground) and $40 \%$ in the swing phase. Each phase is in turn divided into smaller components.

The stance phase begins with the heel strike, where the posterolateral heel first strikes the ground. The foot then de-rotates into 'foot flat'. Mid-stance occurs when both feet are on the ground; and toe-off, with propulsion of the individual, marks the end of the stance phase. The swing phase (which corresponds to single stance phase in the contralateral lower limb) consists of acceleration, whereby the leg swings forward; mid-swing, where the leg clears the ground; and deceleration, in preparation for the heel strike. ${ }^{2}$ Key factors assessed in stance are balance, posture and base of support, while during the swing phase, one should observe cadence, stride length and foot clearance over the ground.

\section{Gait assessment}

\section{History}

The assessment of a patient begins with the history, focusing on complaints of pain, subjective gait abnormalities and reported higher-level mobility task function, such as jumping and running. It is important to ascertain the number of falls in the preceding 12 months. A fall can be defined as unintentional movement from a higher to a lower level, typically rapidly and without control. In the older adult population, the occurrence of more than three falls per year is unacceptable and warrants further investigation.

Patients should be asked about the use of walking aids: duration of use, handedness, compliance with the 'prescription', reason for use and the use of any prosthetics or orthotics.

\section{Examination}

Prior to examination of the gait, one should examine the patient's orthotics, 
prosthetics and footwear to assess wear patterns, which may be pathognomonic. Gait assessment begins as the patient stands to leave the waiting room. This is an opportunity to note any deformities that may be affecting the gait and ascertain which phase of the gait cycle is affected.

Assess the sit-to-stand transfer from a chair and stand-to-sit transfer onto the examination bed. Examine for leg length discrepancy (true and apparent), balance when sitting in comparison to standing, and static balance in comparison to dynamic balance. Complete the assessment with a thorough neurological and joint examination of the patient's upper and lower limbs, including closely supervised heel walking, toe walking, tandem gait and Romberg's test. Romberg's test is useful in patients whose gait appears normal or only mildly abnormal, but it should be performed with caution in those whose gait is already very unsteady, as they are at risk of falling. Romberg's test is primarily a test of proprioception and will be impaired in patients with sensory ataxia, for example, due to a peripheral neuropathy.

Tandem gait is a stringent test of balance that, in the presence of normal power, can indicate evidence of ataxia (cerebellar or sensory).

One should adopt a systematic approach to gait examination (eg observation from head to foot), with adequate visualisation from anterior, posterior and lateral aspects. Table 1 shows the common factors to consider when examining gait.

\section{Common gait abnormalities}

\section{Antalgic gait}

Patients commonly present to general practice with this gait disorder, and it is often referred to colloquially as 'limping'. It is characterised by a shortened single stance phase on the affected side and a secondary rapid swing phase with the contralateral leg to minimise loading through the affected lower limb. It is usually due to pain in the lower limb, and management involves investigating and treating the underlying cause. ${ }^{3}$
A gait aid, such as a walking stick or elbow crutch, may be prescribed. This should be used in the upper limb contralateral to the painful lower limb, as the contralateral upper and lower limb pair move forward in unison during the gait cycle. This motion is partly a vestigial remanent of our quadruped past, but the contralateral upper limb movement also increases the energy efficiency of walking and provides a counterbalance to lower body movement. Arm swing during mobility is controlled by the red nucleus in the midbrain.

\section{Trendelenburg gait}

This gait disorder is often colloquially referred to as a 'waddling' gait, particularly if bilateral. During the single stance phase of the normal gait cycle, there is usually slight inferior pelvic tilt on the weight-bearing leg and slight superior pelvic tilt on the non-weight bearing side to move the body's centre of gravity towards the centre of the weightbearing hip joint, which is essential for balance in the coronal plane. This physiological pelvic tilt also facilitates foot clearance during the swing phase of the gait cycle. ${ }^{3}$

With a Trendelenburg gait, the opposite occurs. This is best summarised by the mnemonic 'sound side sags'. It is usually due to hip abductor weakness (gluteus medius, gluteus minimus and tensor fascia lata). This muscle group usually contracts to stabilise the pelvis during loading at single stance phase. Weakness and wasting of the hip abductors may be related to L4-S1 nerve root lesions or damage to the superior gluteal nerve. The latter may be iatrogenic, for example, during hip arthroplasty.

Occasionally, a Trendelenburg gait may be secondary to a deficient hip joint fulcrum (eg if the hip joint is chronically dislocated, if there is non-union or mal-union of a previous hip fracture, or following a Girdlestone excision arthroplasty).

Management involves targeted physiotherapy to strengthen this muscle group as well as investigation and management of the underlying problem.

\section{Upper motor neuron, dystonic and lower motor neuron gait patterns}

These gait patterns are distinguishable because of changes in muscle tone, with upper motor neuron lesions characterised by a spastic or hypertonic gait. Dystonias usually have an extrapyramidal aetiology, while lower motor neuron lesions are characterised by hypotonia, hyporeflexia, muscle weakness and wasting, with fasiculations.

\section{Hemiplegic gait}

The features of this gait are due to pyramidal weakness and hypertonicity in the affected lower and upper limb, often secondary to an upper motor neuron lesion from a cerebrovascular accident in the contralateral cerebral hemisphere, affecting the corticospinal tract. ${ }^{4}$ The dynamic spasticity with locomotion is often greater than the static spasticity at rest, and patients with underlying weakness may rely on this 'spastic splint' to weight bear. Overtreatment of their spasticity, in the presence of weakness, may paradoxically restrict weight bearing and locomotion, and increase the risk of falls.

Common features include spastic equinovarus deformity at the foot and ankle, reflecting the dominant strength of these muscle groups over their antagonists. Unlike the flaccid plantarflexion seen with a 'foot drop', this is an active state, driven by high tone, that also limits compensatory manoeuvres to obtain foot clearance, such as increased hip and knee flexion. As our legs are the same length, we must functionally shorten our swinging lower limb to avoid forefoot ground contact at mid-swing. We achieve this by 'triple flexion' (ie hip and knee flexion and ankle dorsiflexion). The hemiplegic patient will instead often compensate to achieve foot clearance via contralateral trunk sway, hip hitching and circumduction. The upper limb may be held adducted at the shoulder and flexed at the elbow, wrist and fingers. However, this may be absent, such as in anterior cerebral artery territory strokes, where there may be no upper limb weakness.

The shoes should be examined for excess wear in the toe box region. Falls, and associated injuries, are common. 


\section{Table 1. Common gait examination findings and aetiologies of pathologic gait}

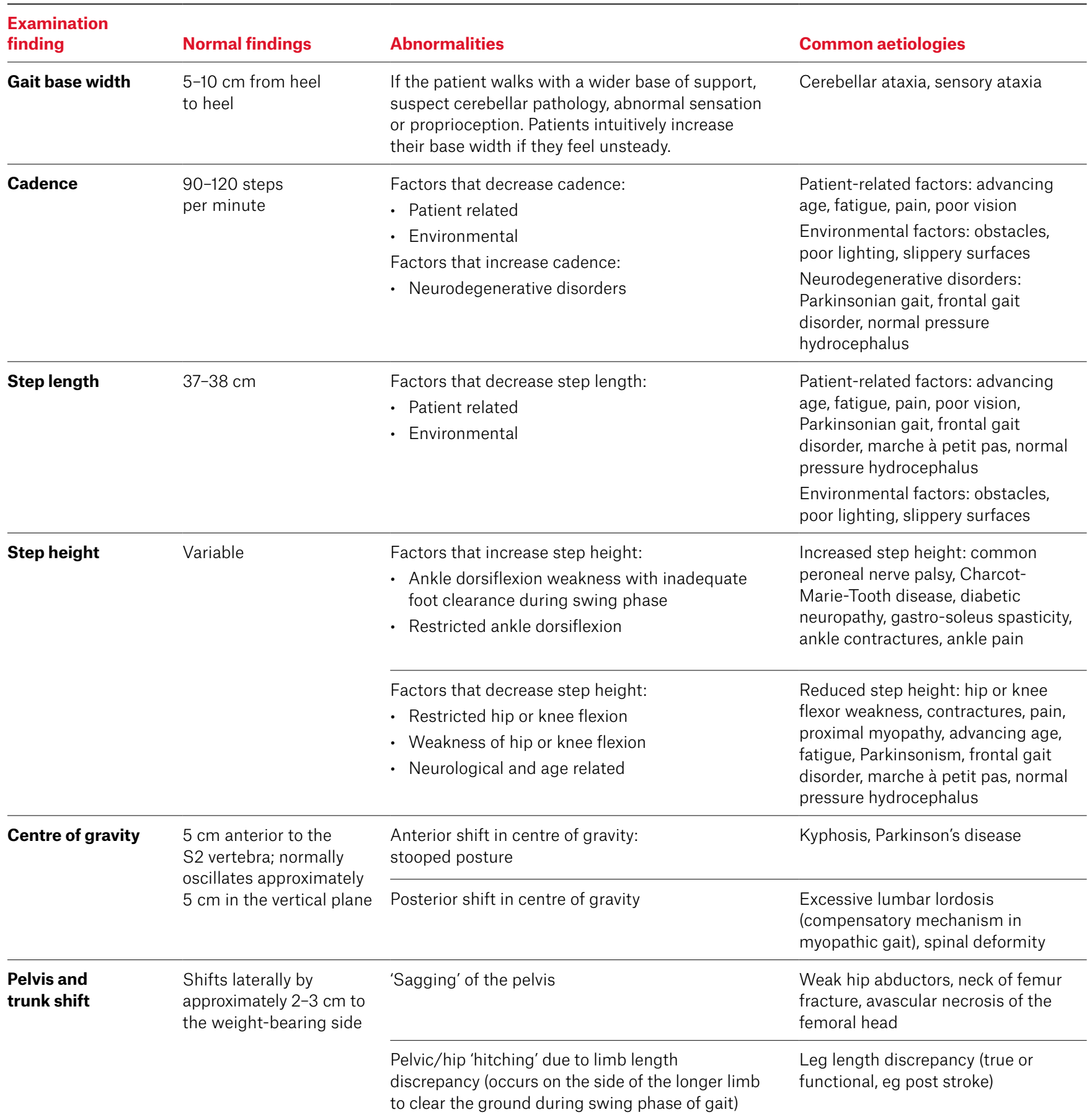

Management is complex and includes treating drivers of spasticity, physiotherapy, walking aids, a single-arm drive wheelchair, orthotics (eg ankle-foot orthoses), custom lycra limb garments, oral antispasticity agents, injectable agents such as phenol or botulinum toxin (by an experienced injector), and surgery in some circumstances.

It is important to consider osteoporosis screening, falls prevention education and balance exercises.
Spastic diplegic gait

This is the typical 'scissoring' gait often seen in spastic cerebral palsy. One to four limbs may be affected. The features of hypertonicity are often similar to those described in spastic hemiplegia, except 
for the disproportionate degree of hip adductor spasticity. Similar signs may be found in patients with paraparesis from incomplete spinal cord lesions above the conus medullaris. Falls are common. ${ }^{4}$

Management principles are similar to those for hemiplegia, with the addition of options, such as obturator nerve blocks, adductor tenotomies, intrathecal baclofen pumps and selective dorsal rhizotomies. Orthopaedic procedures, such as tendon transfers, should generally be combined with measures to treat the underlying spasticity, otherwise new deformities may arise.

\section{Dystonic gait}

Dystonias may be focal or generalised, primary or secondary to limb trauma, or occur as a side effect of treatment for Parkinson's disease.

Dystonic gait disorders frequently appear bizarre, particularly because activity increases dystonic posturing. The abnormal posture of the foot in a dystonic gait typically involves inversion, plantar flexion and tonic extension of the big toe. In many patients, complex types of walking, such as walking backwards and running, are paradoxically less impaired than walking forward and may appear completely unaffected. Some patients will use a sensory 'geste' that helps them manage their symptoms, for example, they are able to walk better with their arm(s) positioned in a certain way.

Referral to a neurologist is advised, as a minority may improve with levodopa; however, the mainstay of treatment is botulinum toxin injection administered by an experienced injector.

\section{Steppage/foot drop gait}

This is characterised by flaccid equinus posturing (ie passive plantar flexion of the foot during the swing phase of the gait cycle). It is sometimes called a 'high stepping gait', referring to the compensatory manoeuvres used to obtain foot clearance during swing phase. ${ }^{5}$ The foot drop presents a trip hazard, and often the patient will intuitively overcompensate to functionally shorten the limb by increasing hip and knee flexion during swing phase. Once this gait has been identified, the clinician should confirm ankle dorsiflexion weakness on bedside examination and look for other signs of a peripheral neuropathy, such as wasting, loss of reflexes or sensory loss in a glove and stocking distribution. While concurrent weakness of ankle eversion is often seen in the setting of peripheral neuropathy (affecting the common peroneal nerve), weakness of ankle inversion may implicate an L4 or L5 radiculopathy.

Peripheral neuropathy is the most common cause: acquired (eg diabetic neuropathy, foraminal impingement of the L4 and/or L5 nerve roots, chronic inflammatory demyelinating polyneuropathy, Guillain-Barré syndrome, motor neuron disease [often mixed upper and lower motor neuron signs], poliomyelitis) or hereditary peripheral neuropathy (eg Charcot-Marie-Tooth disease).

Management involves targeted physiotherapy to strengthen the peroneal muscle compartment, provision of an ankle foot orthosis, and investigations (electromyography and nerve conduction studies for a peripheral neuropathy and neuroimaging if a radiculopathy is suspected), followed by management of the underlying problem.

\section{Flaccid paralytic gait}

This pattern results from proximal lower limb weakness (eg involving the knee extensors) due to a lower motor neuron lesion such as poliomyelitis. The patient will often intuitively adopt knee hyperextension (genu recurvatum), with the force vector, at initial ground contact, directed anterior to the knee joint to prevent the knee collapsing into flexion during maximal loading at the single stance phase of the gait cycle. Provision of a knee-ankle-foot orthosis may be required.

\section{Parkinsonian gait}

This is characterised by a stooped posture, with decreased gait speed, step height and length. It is often referred to as a slow, shuffling gait. The trunk and lower extremities are usually flexed and the patient appears to be 'chasing their own centre of gravity', which is transposed forward by truncal flexion. Festination may be evident from the history or on examination and describes an involuntary gait pattern, with shortened step length, in which the steps become progressively more rapid as the patient attempts to 'catch' their forward-shifting centre of gravity, though often forward progression is decreased overall.

Other prominent features include: hypokinesia, bradykinesia, freezing (often with gait initiation, turns and thresholds), increased postural sway, propulsion, retropulsion, reduced arm swing and 'en bloc' turning wherein patients take multiple small steps to accomplish a turn. Instead of the usual heel strike, patients may have initial ground contact at the midfoot or forefoot. Falls are common. ${ }^{6}$

Management involves targeted physiotherapy, correction of the patient's posture and gait, prescription of walking aids, and medication management of the patient's Parkinsonian symptoms and signs, the discussion of which is beyond the scope of this paper. Sensory cuing (eg visual cues such as stripes on the floor, a metronome or 'marching' music) may be helpful for freezing; a feature of Parkinsonism for which pharmacotherapies are often ineffective.

Severe, fixed, forward flexion of the trunk is referred to as camptocormia. This may be due to Parkinson's disease, dystonia, medication side effects, spinal disc herniation, severe spondylosis or previous spinal trauma.

\section{Frontal gait disorder}

Affected individuals are usually elderly; they experience difficulty with gait initiation and mobilise with short shuffling steps on a broad base. This may be described as a 'marche à petit pas'. Arm swing is usually normal. Frontal gait disorder may be may be mistaken for a Parkinsonian gait; however, there are no upper body Parkinsonian symptoms. It is sometimes referred to as 'lower body Parkinsonism' but is rarely dopamine responsive. Falls are relatively common. Causes include small vessel cerebrovascular disease resulting in deep white matter ischaemia, ${ }^{7}$ dementia and normal pressure hydrocephalus.

Management involves physiotherapy and walking aid prescription. 


\section{Normal pressure hydrocephalus}

A subtype of frontal gait disorder, this classically presents with the triad of cognitive impairment, urinary incontinence and gait impairment. The gait is usually slow, shuffling and broad based, with the feet appearing 'stuck to the floor'. Some gait features may appear Parkinsonian. ${ }^{4}$

Management involves walking aid prescription and referral to a neurosurgeon for consideration of cerebrospinal fluid diversion (eg ventriculoperitoneal shunt insertion).

\section{Ataxic gaits}

These are typically broad-based gaits, involving uncoordinated muscle activity, dysmetria and trunk titubation. The causes of ataxia are many and varied but may be broadly divided into cerebellar, vestibular and sensory types. There may be associated upper limb and ocular signs. Falls are common. ${ }^{8}$

Once a broad-based ataxic gait has been identified, it is critical to distinguish between a cerebellar or sensory ataxia. This is done at the bedside by studying the gait pattern and assessing for concomitant signs of cerebellar disease (intention tremor, dysmetria, dysdiadochokinesia, nystagmus and dysarthria), or evidence of a sensory neuropathy/neuronopathy (loss of proprioception, hyporeflexia, positive Romberg's test). Cerebellar and sensory ataxia may co-exist, for example, in the setting of chronic alcohol overuse or cerebellar ataxia, neuropathy and vestibular areflexia syndrome (CANVAS).

\section{Sensory ataxia}

Sensory ataxia presents as a broad-based, unsteady gait, reliant on visual cues to assist stability; thus, it is often significantly worse in the dark. There is dysmetria and often heavy ground contact at the start of single stance phase. It is due to lower limb sensory impairment secondary to peripheral neuropathy, dorsal root or dorsal column lesions in the spinal cord. ${ }^{4}$

\section{Cerebellar ataxia}

Cerebellar ataxia presents similarly as a broad-based gait; however, the patient is unable to rely on visual cues because of cerebellar dysfunction. The patient will not be able to tandem walk, and Romberg's test is negative. There may be truncal instability if the cerebellar vermis is involved.

Management of ataxia involves investigation and management of the underlying pathology, where possible, and provision of a walking aid, as this may assist in providing tactile cues and a broader base of support, to assist stability. The practitioner can also consider osteoporosis screening, falls prevention education and balance exercises.

\section{Myopathic gait}

Truncal and proximal myopathy results in a difficult and slow sit-to-stand transfer, often using the upper limbs to elevate the trunk by 'walking' the hands up the thighs. This is commonly due to muscular dystrophy. Patients with myopathy may have difficulty rising from a chair, which can be a clue in the waiting room. They stand with an exaggerated lumbar lordosis which, in combination with the weak abdominal musculature, results in a protuberant abdomen. Such patients display a broad-based, bilateral Trendelenburg gait. Falls are relatively common. ${ }^{9}$

Management involves prescription of walking aids; physiotherapy is of limited use. A wheelchair may be required for longer distances and as the disease progresses.

\section{Complex gaits}

These have a variable combination of features from the aforementioned gait patterns, secondary to coexisting pathologies or patterns that have been modified by medical intervention. Patients with a history of childhood polio may also display a complex gait pattern, such as lower motor neuron weakness affecting the entire lower limb, including genu recurvatum and shortening secondary to lower limb hypoplasia. ${ }^{2}$

Management involves investigation of the underlying causes and prescription of gait aids; wheelchairs may be required for longer distances.

\section{Short-leg gait}

Short-leg gait is due to either true leg length inequality - 'short side sags' - or apparent shortening, secondary to pelvic obliquity or scoliosis. A shoe raise (internal and/or external), together with a gait aid, may be required.

\section{Gait arrhythmia}

Gait variability may be a marker of Alzheimer's disease. A high degree of variability, characterised by 'strideby-stride fluctuations in distance and time', is associated with lower cognitive performance and may help to differentiate between Alzheimer's dementia and other neurodegenerative disorders. ${ }^{10}$

\section{Less common gait patterns}

Less common gait patterns include a stiff-leg gait (eg following a knee arthrodesis, which may require a contralateral shoe raise to facilitate foot clearance) and hyperkinetic gait disorders (eg in athetoid cerebral palsy or Huntington's disease).

\section{Functional gait disorders}

Functional gait disorders should remain a 'diagnosis of exclusion'. Patients with functional gait disorders may display an elaborate gait, without any clear pattern or repetition throughout the gait cycle. Although there are often multiple 'near falls' and a bizarre gait pattern, patients rarely fall, as a result of preserved higher order balance function. Patients may be able to run, hop or walk backwards without falling (caution must be taken, however, as this can also be seen in dystonic gaits). There may be 'giving way' when assessing lower limb power on manual muscle testing. Hoover's test may be helpful in supporting the diagnosis. ${ }^{2}$

Periodic review is advised, as a minority of patients will subsequently be found to have a related organic disorder. Consultation with a psychiatrist may be appropriate.

\section{Conclusion}

In summary, it is important to assess a broad range of cues including posture, walking aids, footwear, orthotics and prosthetics. The gait cycle can be broadly divided into the stance and swing phases. It is important to assess the width of the 
base of gait, step length, step height, cadence, centre of gravity and trunk shift during the gait cycle and observe for characteristic patterns. Gait examination, from the time the patient is called from the waiting room, will help the clinician focus on the relevant systems to examine, investigate and treat.

Emerging trends include the use of wearable devices for continuous monitoring of gait as an additional approach to quantitatively assess mobility in real-life settings. ${ }^{11}$

\section{Authors}

Appukutty Manickam MBBS, MRCS (Ed), MMed (Gen Surg), Advanced Trainee, Rehabilitation Medicine, Calvary Hospital, Kogarah, NSW Matthew D Gardiner MBBS (Hons I), FRACGP, FAFRM (RACP), Senior Staff Specialist, Rehabilitation Medicine, Calvary Hospital, Kogarah, NSW; Conjoint Lecturer, Faculty of Medicine, University of NSW, Sydney, NSW
Competing interests: None.

Funding: None.

Provenance and peer review: Not commissioned, externally peer reviewed.

\section{Correspondence to:}

matthew.gardiner@health.nsw.gov.au

\section{References}

1. Cappozzo A. Considerations on clinical gait evaluation. J Biomech 1983;16(4):302. doi: 10.1016/0021-9290(83)90202-6.

2. Cooper RA, Quatrano LA, Stanhope SJ, et al. Gait analysis in rehabilitation medicine: A brief report. Am J Phys Med Rehabil 1999;78(3):278-80. doi: 10.1097/00002060-199905000-00019.

3. Della Croce U, Leardini A, Chiari L, Cappozzo A. Human movement analysis using stereophotogrammetry. Part 4: Assessment of anatomical landmark misplacement and its effects on joint kinematics. Gait Posture 2005;21(2):226-37. doi: 10.1016/j. gaitpost.2004.05.003.

4. Gilman S, Winans Newman S. Manter and Gatz's essentials of clinical neuroanatomy and neurophysiology. 10th edn. Philadelphia, US: FA Davis Company, 2003.

5. Keenan MA, Lee GA, Tuckman AS, Esquenazi A. Improving calf muscle strength in patients with spastic equinovarus deformity by transfer of the long toe flexors to the Os calcis. J Head Trauma Rehabil 1999;14(2):163-75. doi: 10.1097/00001199199904000-00006.

6. Samii A, Nutt JG, Ransom BR. Parkinson's disease. Lancet 2004;363(9423):1783-93. doi: 10.1016/ S0140-6736(04)16305-8.

7. Tyrrell PJ. Apraxia of gait or higher level gait disorders: Review and description of two cases of progressive gait disturbance due to frontal lobe degeneration. J R Soc Med 1994;87(8):454-56.

8. Mayo Clinic Department of Neurology. Mayo Clinic examinations in neurology. 7th edn. St Louis, US: Mosby, 1998.

9. Campbell W, editor. Dejong's the neurological examination. 6th edn. Philadelphia, US: Lippincott Williams and Wilkins, 2005.

10. Pieruccini-Faria F, Black SE, Masellis M, et al. Gait variability across neurodegenerative and cognitive disorders: Results from the Canadian Consortium of Neurodegeneration in Aging (CCNA) and the Gait and Brain Study. Alzheimers Dement 2021;17(8):1317-28. doi: 10.1002/alz.12298.

11. Hausdorff J. Early motor signal specific to Alzheimer's identified. Medscape Medical News. 25 February 2021. Available at www.medscape.com/ viewarticle/946453 [Accessed 12 October 2021]. 